\title{
NIL DERIVATIONS AND CHAIN CONDITIONS IN PRIME RINGS
}

\author{
L. O. CHUNG AND Y. KOBAYASHI
}

\begin{abstract}
It is known that in a prime ring, a derivation nilpotent on a nonzero ideal must also be nilpotent in the entire ring. In this paper we show that a derivation of a prime ring is not necessarily nil even though it is nil on a nonzero ideal. It is nil if the ring satisfies the ascending chain condition on left (right) annihilator ideals.
\end{abstract}

Let $R$ be a ring and $\partial$ a derivation of $R$. Then $\partial$ is called nil if for any $x \in R$ there is a positive integer $n=n(x)$ such that $\partial^{n} x=0$ (see [2]). Here, if the number $n$ can be taken independently of $x, \partial$ is called nilpotent. Assume that $R$ is a prime ring and $I$ is a nonzero ideal of $R$. Chung and Luh [3] proved that if $\partial$ is nilpotent on $I$, then it is nilpotent also on $R$. Nil derivations are important because they are related to automorphisms of the ring via the exponential mapping [2]. The purpose of this paper is to answer the following

Question (*). Let $\partial$ be a derivation on a prime ring $R$. If $\partial$ is nil on a nonzero ideal $I$ of $R$, is it nil on the whole ring $R$ ?

The answer is negative, as will be shown in $\$ 1$. We shall give three counterexamples of independent interest. In $\$ 2$ we show that the answer is positive under some additional conditions, especially under certain chain conditions.

We would like to express our thanks to Professor J. Luh and the referee for many valuable suggestions. For instance, Example 2 in $\$ 1$ was suggested by the referee.

1. Examples. Throughout this section $p$ is a prime number. For a subset $X$ of a ring $R$ with $1, X^{-1}$ denotes the set of inverses of all invertible elements of $X$.

LEMMA 1. Let $R$ be a ring with 1 of characteristic $p$ and $X$ a subset of $R$. If $a$ derivation $\partial$ of $R$ is nilpotent (resp. nil) on $X$, then it is nilpotent (resp. nil) on the subring of $R$ generated by $X \cup X^{-1}$.

Proof. Let $a, b, c \in R$ and suppose $a b=c$. Then for any positive integer $n$, we have

$$
\left(\partial^{p^{\prime \prime}} a\right) b+a \partial^{p^{n}} b=\partial^{p^{n}} c .
$$

So, if $\partial^{p^{n}} a=\partial^{p^{n}} b=0$, then $\partial^{p^{n}} c=0$; and if $\partial^{p^{n}} b=\partial^{p^{n}} c=0$ and $b$ is invertible, then $\partial^{p^{n}} a=0$. From this observation the assertion of the lemma follows.

Received by the editors March 22, 1984 and, in revised form, August 9, 1984.

1980 Mathematics Subject Classification. Primary 16A72.

Key words and phrases. Nil derivation, prime ring, ascending chain condition. 
If we only consider the subring generated by $X$ in Lemma 1 , the existence of 1 in $R$ is not necessary. The lemma enables us to construct a derivation on a field of positive characteristic, which is nil but not nilpotent.

EXAMPLE 1. Let $K$ be a field of positive characteristic. On the (commutative) polynomial ring $K\left[x_{1}, x_{2}, \ldots\right]$ over $K$ in a countable number of variables, define a derivation $\partial^{\prime}$ over $K$ by $\partial^{\prime} x_{i}=x_{i-1}$ for $i \geqslant 2$ and $\partial^{\prime} x_{1}=0$. $\partial^{\prime}$ can be extended uniquely to a derivation $\partial$ of the quotient field $K\left(x_{1}, x_{2}, \ldots\right)$. Then $\partial$ is nil by Lemma 1 but not nilpotent.

The reason why we need an infinite number of variables in Example 1 can be explained by the following proposition. By a right quotient ring of $R$ we mean a ring $S$ with 1 containing $R$ as it subring such that any element $x \in S$ is written $x=a b^{-1}$ for some $a, b \in R$ with $b$ invertible in $S$.

Proposition 1. Let $R$ be a finitely generated algebra over a commutative ring $K$ of characteristic $p$. Then a nil derivation of any right quotient ring $S$ of $R$ over $K$ is nilpotent.

Proof. Let $R$ be generated by a finite set $X$. A nil derivation $\partial$ of $S$ over $K$ is nilpotent on $X \cup K$. So it is nilpotent on $R$ and, consequently, nilpotent on $S$ by Lemma 1.

A derivation $\partial$ of $R$ is called locally nilpotent if for any $a \in R$ there is a subring $S$ of $R$ such that $a \in S, \partial S \subseteq S$, and $\partial$ is nilpotent on $S$.

Proposition 2. Let $R$ be a ring of characteristic $p$. Then a nil derivation of $R$ is locally nilpotent.

Proof. Let $\partial$ be a nil derivation of $R$ and let $x \in R$. Then $\partial^{n} x=0$ for some positive integer $n$. Let $S$ be the subring generated by $\left\{x, \partial x, \ldots, \partial^{n-1} x\right\}$. Then $\partial S \subseteq S$ and $\partial$ is nilpotent on $S$ by Lemma 1 .

In Propositions 1 and 2 the positivity of the characteristic is essential. In fact, let $R=K[x]$ be the polynomial ring over a field $K$ of characteristic 0 , and let $\partial$ be the ordinary derivation given by $\partial^{n} x=n x^{n-1}$. Then $\partial$ is nil but not locally nilpotent. The following example answers Question (*) in the negative in the case of positive characteristic.

EXAmple 2. Let $F=K\left(x_{1}, x_{2}, \ldots\right)$ be the field and $\partial$ the derivation of $F$ given in Example 1. Let $R$ be the ring of countable by countable, row by column finite matrices over $F$. Then $R$ is a prime ring with the unique proper ideal $I$ consisting of matrices with only finitely many nonzero entries. Define a derivation $\bar{\partial}$ of $R$ by $\bar{\partial} A=\left[\partial a_{i j}\right]$ for a matrix $A=a_{i j}$ in $R$. Then $\bar{\partial}$ is nil on the ideal $I$ but not nil on the whole ring $R$.

The following proposition shows that the construction in Example 2 does not work in the case of characteristic 0 .

Proposition 3. A division ring $D$ of characteristic 0 has no nonzero nil derivation.

Proof. Suppose $D$ has a nonzero nil derivation $\partial$. Then there is $a$ in $D$ such that $\partial a \neq 0$ and $\partial^{2} a=0$. Let $b$ be the inverse of $a$; then $\partial^{n-1} b \neq 0$ and $\partial^{n} b=0$ for some 
positive integer $n$. We have $0=\partial^{n}(1)=\partial^{n}(a b)=n(\partial a) \partial^{n-1} b$, but this is a contradiction.

The following example covers the case of characteristic 0 .

EXAMPLE 3. Let $K$ be a field of characteristic 0 , and let $S=K\langle x, y, z\rangle$ be the polynomial ring in three noncommuting variables $x, y$, and $z$ over $K$. For a monomial $M$ in $S, \operatorname{deg}_{x} M$ and $\operatorname{deg}_{y} M$ denote the degree of $M$ with respect to $x$ and $y$, respectively. Let $I$ be the ideal of $S$ generated by the monomials of the forms $M z$ and $z M$, where $M$ ranges over all the monomials in $x$ and $y$ such that $\operatorname{deg}_{x} M>$ $\operatorname{deg}_{x} M$. Define a derivation $\partial$ of $S$ over $K$ by $\partial x=x^{2}, \partial y=1$, and $\partial z=0$. Since $\partial I \subseteq I, \partial$ induces a derivation $\bar{\partial}$ of the factor ring $R=S / I$. Then $R$ is a prime ring and $\partial$ is nil on the ideal $R \bar{z} R$ but not nil on $R$, where $\bar{z}$ is the residue class of $z$ modulo $I$.

The algebra $R$ in Example 3 is finitely generated. In view of Proposition 1, it seems difficult to construct such an example in the case of a positive characteristic. The following example works for arbitrary characteristic.

EXAMPLE 4. Let $K$ be a field and $R$ the ring of countable by countable, row by column finite matrices over $K$. Let $A$ be a matrix in $R$ formally written in the following way:

$$
A=\sum_{i=1}^{\infty} e(i, i+1)-\sum_{i=1}^{\infty} e\left(i^{2}, i^{2}+1\right),
$$

where $e(i, i+1)$ is the matrix with 1 in the $(i, i+1)$ th place and 0 elsewhere. Then the inner derivation $\mathrm{ad}_{A}$ induced by $A$ is nil, not on $R$, but on the unique proper ideal consisting of matrices with only finitely many nonzero entries.

2. Chain conditions. The rings given in Examples $2-4$ do not satisfy the ascending (descending) chain condition on one-sided ideals. In this section we give some positive results on Question (*) under certain conditions.

THEOREM 1. Let $R$ be a ring, $I$ a right ideal of $R$, and $\partial$ a derivation of $R$. If $\partial$ is nil on $I$, then for any $a \in I$ and $x \in R$ there is a positive integer $N=N(a, x)$ such that $a \partial^{n} x=0$ for all $n \geqslant N$.

Proof. If we let $J=\sum_{i=0}^{\infty} \partial^{i} I$, then $J$ is a right ideal of $R$ such that $I \subseteq J, \partial J \mid J$, and $\partial$ is nil on $J$. So we may assume from the beginning that $\partial I \subseteq I$. We prove the assertion by induction on the nilpotency $m$ of $\partial$ on $a$, that is $\partial^{m} a=0$, but $\partial^{m-1} a \neq 0$. If $m=0$, then $a=0$ and there is nothing to prove. Let $m>0$. Since $\partial^{i} a$ is in $I$ and its nilpotency is smaller than that of $a$ for $i=1, \ldots, m-1$, the induction hypothesis assures us that there is a positive integer $N_{1}=N_{1}(a, x)$ such that $\left(\partial^{i} a\right) \partial^{n} x=0$ for $i=1, \ldots, m-1$ and $n \geqslant N_{1}$. Since $a x \in I$, we see $\partial^{N_{2}}(a x)=0$ for some positive integer $N_{2}=N_{2}(a, x)$. Therefore, if $n \geqslant \max \left\{N_{2}, N_{1}+m-1\right\}$, then

$$
0=\partial^{n}(a x)=\sum_{i=0}^{n}\left(\begin{array}{c}
n \\
i
\end{array}\right)\left(\partial^{i} a\right) \partial^{n-i} x=a \partial^{n} x
$$

This completes the proof. 
COROLLARY. Let $I$ be a right ideal of $R$ containing at least one right regular element. Then a derivation of $R$ which is nil on $I$ is nil on $R$.

THEOREM 2. Let I be a nonzero right ideal of a prime ring $R$ which satisfies the ascending chain condition ( $A C C$ ) on left annihilator ideals. Then a derivation of $R$ which is nil on I is nil on $R$.

Proof. Let $\partial$ be a derivation of $R$ which is nil on $I$. Let $x \in R$ and let $n$ be a nonnegative integer. Define

$$
I_{n}(x)=\left\{a \in R \mid a \partial^{i} x=0 \text { for all } i \geqslant n\right\} .
$$

Then we have an ascending chain $\left\{I_{n}(x)\right\}_{n=0,1,2 \ldots}$ of left annihilator ideals of $R$. By assumption there is a positive integer $N$ such that $I_{N}(x)=I_{N+1}(x)=\cdots$. By Theorem 1 we find that

$$
I \subseteq \bigcup_{n=0}^{\infty} I_{n}(x)=I_{N}(x)
$$

This means $I \partial^{N} x=0$, and, consequently, $\partial^{N} x=0$ because $R$ is prime.

COROllaRY 2. Let $R$ be a prime right (left) Goldie ring. If a derivation $\partial$ of $R$ is nil on a nonzero right ideal of $R$, then $\partial$ is nil on $R$.

Proof. Since, in either case, $R$ satisfies the ACC on left annihilators $[1,1.15]$, the conclusion follows from Theorem 2 .

Theorem 2 is not true at all if we replace "prime" by "semiprime". In fact, let $R_{1}$ and $R_{2}$ be prime rings and $\partial_{1}$ a nonnil derivation of $R_{1}$. The derivation $\partial$ of $R=R_{1} \oplus R_{2}$, defined by $\partial\left(x_{1}+x_{2}\right)=\partial x_{1}, x_{i} \in R_{i}$, is nil, not on $R$, but (actually zero) on the ideal $R_{2}$. So the conditions in the next theorem may be reasonable.

THEOREM 3. Let $R$ be a ring with the ACC on left annihilator ideals. Let $I$ be a right ideal of $R$ containing the prime radical of $R$. If a derivation $\partial$ of $R$ is nil on $I$, then it is nil on the ideal $\bar{I}$ generated by $I$. If, moreover, $\bar{I}$ is essential in $R$ as a left ideal, then $\partial$ is nil on $R$.

Proof. We may assume $\partial I \subseteq I$. Then $\partial \bar{I} \subseteq \bar{I}$. The prime radical $P$ of $R$ is an intersection of prime ideals $P_{\alpha}$ of $R$. Let $x \in \bar{I}$; then by the proof of Theorem 2, $I \partial^{n} x=0$ for some positive integer $n$. If $I \nsubseteq P_{\alpha}$, then $\partial^{n} x \in P_{\alpha}$. On the other hand, if $I \subseteq P_{\alpha}$, then $\partial^{n} x \in \bar{I} \subseteq P_{\alpha}$. It follows that $\partial^{n} x \in P$. Since $\partial$ is nil on $P$ by assumption, it is nilpotent on $x$.

Now let $x$ be an arbitrary element in $R$. Since $I \partial^{n} x=0, \bar{I} \partial^{n} x=0$ holds. So, if $\bar{I}$ is an essential left ideal, then $\partial^{n} x$ is in the left singular ideal $Z$ of $R$. Since $Z$ is a nilpotent ideal by $[1,1.6]$, we find that $\partial^{n} x \in Z \subseteq P \subseteq I$. Thus, $\partial$ is nilpotent on $x$.

In Theorems 2 and 3 the ACC on left annihilator ideals is indispensable. It cannot be replaced even by the ACC on right annihilator ideals, as the following example shows.

EXAMPLE 5. Let $K$ be a field of arbitrary characteristic. Let $R$ be the factor ring of the polynomial ring $K\left\langle x_{1}, y_{1}, x_{2}, y_{2}, \ldots\right\rangle$ over $K$ in a countable number of noncommuting variables $x_{1}, y_{1}, x_{2}, y_{2}, \ldots$ modulo the ideal $I$ generated by all $x_{i} y_{j}$ and 
$y_{i} y_{j}-y_{j} y_{i}$ such that $i<j$. Define a derivation $\partial$ of $R$ over $K$ by $\partial x_{i}=x_{i-1}$ for $i \geqslant 2$, $\partial x_{1}=0$, and $\partial y_{j}=y_{j+1}$ for $j \geqslant 1$ modulo $I$. Then $R$ is a prime ring with the ACC on right annihilator ideals, $\partial$ is nil on the right ideal $\sum_{i=1}^{\infty} \bar{x}_{i} R$, but it is not nil on every nonzero left ideal. In fact, for any nonzero element $\bar{z}$ in $R, \partial$ is not nilpotent on $\bar{y}_{j} \bar{z}$.

Finally, the following example shows that, in Theorem 3, the condition that $I$ contain the prime radical of $R$ is also necessary.

EXAMPLE 6. Let $K$ be a field and let

$$
R=\left\{\left[\begin{array}{lll}
a & c & d \\
0 & a & 0 \\
0 & 0 & b
\end{array}\right] \mid a, b, c, d \in k\right\} .
$$

Then $R$ is an Artinian ring whose prime radical is

$$
\left[\begin{array}{lll}
0 & K & K \\
0 & 0 & 0 \\
0 & 0 & 0
\end{array}\right]
$$

The derivation $\partial$ defined by

$$
\partial\left[\begin{array}{lll}
a & c & d \\
0 & a & 0 \\
0 & 0 & b
\end{array}\right]=\left[\begin{array}{lll}
0 & 0 & d \\
0 & 0 & 0 \\
0 & 0 & 0
\end{array}\right]
$$

is zero on the right ideal

$$
I=\left[\begin{array}{lll}
0 & K & 0 \\
0 & 0 & 0 \\
0 & 0 & K
\end{array}\right]
$$

of $R$ but not nil on the ideal

$$
\bar{I}=\left[\begin{array}{lll}
0 & K & K \\
0 & 0 & 0 \\
0 & 0 & K
\end{array}\right]
$$

generated by $I$, and $\bar{I}$ is an essential left ideal of $R$.

NOTE ADDED IN PROOF. Related and interesting results were also obtained independently by Y. Hirano and H. Yamakawa in a paper On nil and nilpotent derivation to appear in Math. J. Okayama Univ.

\section{REFERENCES}

1. A. W. Chatters and C. R. Hajarnavis, Rings with chain conditions, Pitman, Boston, Mass., 1980.

2. L. O. Chung, Nil derivations, J. Algebra (to appear).

3. L. O. Chung and J. Luh, Nilpotent derivations in an ideal, Proc. Amer. Math. Soc. 90 (1984), 211-214.

Department of Mathematics, North Carolina State University, Raleigh, North Carolina $27695-8205$

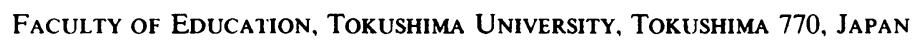

